\title{
Molnupiravir in unvaccinated patients with COVID-19
}

Overview of: Jayk Bernal A, Gomes da Silva MM, Musungaie DB, et al. Molnupiravir for oral treatment of COVID-19 in nonhospitalized patients. NEJM. 2021;doi:10.1056/NEJMoa2116044 [Epub ahead of print 16 Dec 2021].

\section{Key learning points}

- Molnupirvair is a prodrug of N-hydroxycytidine, which is active against RNA viruses including SARS-CoV-2.

- Molnupiravir was compared with placebo in non-hospitalised unvaccinated adults with mild-to-moderate COVID-19 and at least one risk factor for severe COVID-19 illness.

- Treatment with oral molnupiravir within 5 days of the start of symptoms reduced the risk of hospitalisation or death.

The full trial data showed that the risk of hospitalisation or death in nonhospitalised unvaccinated adults with mild-to-moderate COVID- 19 was $6.8 \%$ with molnupiravir and $9.7 \%$ with placebo. ${ }^{1}$

\section{Overview}

This double-blind randomised placebocontrolled trial evaluated the efficacy and safety of molnupiravir started within 5 days of onset of signs or symptoms in non-hospitalised unvaccinated adults with mild-to-moderate laboratory-confirmed COVID-19 and who were at risk of severe COVID-19 illness. ${ }^{1}$ Participants had at least one risk factor for development of severe COVID-19 (eg, age $>60$ years, active cancer, chronic kidney disease, obesity, diabetes mellitus). Exclusion criteria included an anticipated need for hospitalisation for COVID-19 within the next 48 hours, estimated glomerular filtration rate $<30 \mathrm{~mL} / \mathrm{min} / 1.73$ $\mathrm{m}^{2}$, pregnancy and SARS-CoV-2 vaccination. Participants were randomised to either oral molnupiravir $800 \mathrm{mg} /$ day or placebo for 5 days. The primary outcome was the incidence of hospitalisation (defined as $\geq 24$ hours of acute care in a hospital) for any cause or death up to day 29.

The full analysis included 1433 participants from 107 sites in 20 countries (median age 43 years, $51 \%$ female), and the most common risk factors included obesity (74\%), age $>60$ years (17\%) and diabetes mellitus (16\%). ${ }^{1}$ The three most common SARS-CoV-2 variants were delta (58\%), mu (21\%) and gamma (11\%). A total of 1408 people were included in the modified intention-to-treat analysis. Those who received molnupiravir had a lower risk of hospitalisation or death compared with placebo $(6.8 \%$ vs $9.7 \%$, absolute difference $2.9 \%, 95 \% \mathrm{Cl}-5.9$ to -0.1$)$. When the data were analysed for only COVID-19-related hospitalisations or deaths, the event rate was $6.3 \%$ in the molnupiravir group and $9.2 \%$ in the placebo group (absolute difference 2.8\%, $95 \% \mathrm{Cl}-5.7$ to 0.0 ).

The proportion of adverse events was similar with $30 \%$ in the molnupiravir group and $33 \%$ in the placebo group. ${ }^{1}$ Commonly reported events that occurred in $\geq 2 \%$ of participants were COVID-19 pneumonia (6.3\% vs $9.6 \%$ ), diarrhoea (2.3\% vs $3.0 \%$ ) and bacterial pneumonia (2.0\% vs $1.6 \%$ ).

(The trial was supported by Merck Sharp and Dohme).

\section{Context}

Interim efficacy results using data from 762 people, of whom 385 received molnupiravir, were issued in a press release. ${ }^{2}$ Hospitalisation or death occurred in $7.3 \%$ of those in the molnupiravir group and $14.1 \%$ of those in the placebo group (number needed to treat [NNT] $=15) .{ }^{12}$ The full results from 1408 people, of whom 716 received molnupiravir, showed a smaller difference in the rate of hospitalisation or death (NNT=35). The reason for the difference between interim and final results is not known. ${ }^{1}$

It is important to note that the trial only included people who had not been vaccinated against COVID-19. ${ }^{13}$ The study's authors commented that they made the choice to include unvaccinated patients in order to focus on those most likely to need antiviral treatment and 'to facilitate more rapid evaluation of the therapeutic efficacy of molnupiravir'. ${ }^{1}$ The US Food and Drug Administration's evaluation report noted that 'while vaccinated individuals were excluded... no treatment benefit was discernible among the small subgroup or participants who were seropositive (due to natural immunity) at baseline given the small number of events overall. However, there are numerous limitations to extrapolating findings from patients with natural immunity to those with vaccine-induced immunity. ${ }^{4}$ In the UK, over $80 \%$ of the population aged $\geq 12$ years has received two doses of COVID-19 vaccine, and it is not known how effective molnupiravir will be in a highly vaccinated population. ${ }^{3}$

\section{Contributors DTB Team.}

Provenance and peer review Commissioned; internally peer reviewed.

(c) BMJ Publishing Group Limited 2022. No commercial re-use. See rights and permissions. Published by BMJ.

\section{References}

1 Jayk Bernal A, Gomes da Silva MM, Musungaie $\mathrm{DB}$, et al. Molnupiravir for oral treatment of Covid-19 in nonhospitalized patients. NEng/ J Med 2021. doi:10.1056/NEJMoa2116044. [Epub ahead of print: 16 Dec 2021].

2 Merck. Merck and Ridgeback's investigational oral antiviral molnupiravir reduced the risk of hospitalization or death by approximately 50 percent compared to placebo for patients with mild or moderate COVID-19 in positive interim analysis of phase 3 study [online], 2021. Available: https://www.merck.com/news/merckand-ridgebacks-investigational-oralantiviral-molnupiravir-reduced-the-risk-ofhospitalization-or-death-by-approximately50-percent-compared-to-placebo-forpatients-with-mild-or-moderat/ [Accessed 11 Jan 2022].

3 Cave JA, Phizackerley D. Molnupiravir: evidence by press release. Drug Ther Bull 2022;60:10.1136/dtb.2021.000064.

4 U.S. Food and Drug Administration Center for Drug Evaluation and Research. FDA briefing document antimicrobial drugs Advisory Committee meeting, 2021. Available: https://www.fda.gov/advisorycommittees/advisory-committee-calendar/ november-30-2021-antimicrobialdrugs-advisory-committee-meetingannouncement-11302021-11302021 [Accessed 11 Jan 2022]. 